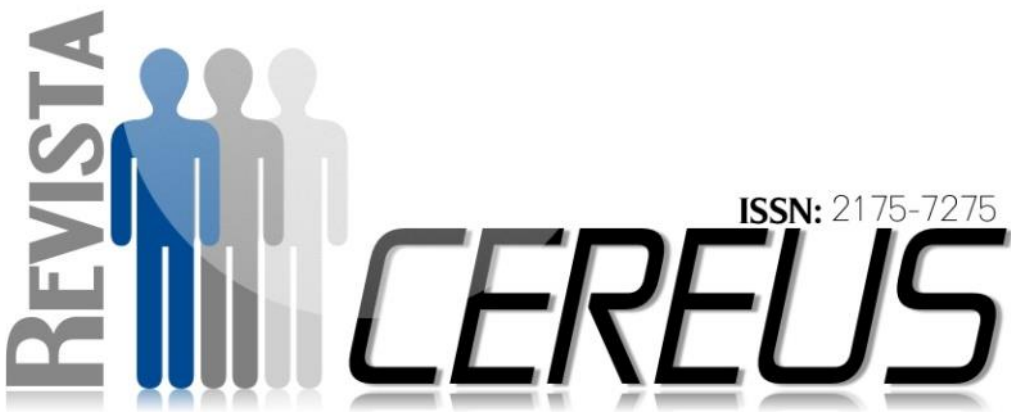

\section{PROPENSÃO À DEPENDÊNCIA DA INTERNET: UM ESTUDO COM ACADÊMICOS DE UM CENTRO UNIVERSITÁRIO}

KLINGER, Ellen Fernanda'; SILVA, Jeann Bruno Ferreira;

MARINHO, Vinícius Lopes; MIRANDA, Karine Wanderley4; REIS, Thais Valadão ${ }^{5}$

\section{RESUMO}

Introdução: o emprego de novas tecnologias para facilitar a maneira de se comunicar das pessoas tem aumentando progressivamente na última década. Em consonância com esse crescimento de amplitude global, o uso excessivo do fenômeno da internet às redes sociais vem ocasionando severas transformações na sociedade, bem como alterações nos hábitos e comportamento dos indivíduos, o que pode caracterizar um quadro de dependência. Objetivo: investigar

1 Doutoranda pelo programa de Psicologia - PUC GO, Mestre em Distúrbios da Comunicação Humana - UFSM. Professora do Centro Universitário Unirg. Email para correspondência: klingerellen@gmail.com

2Psicólogo. Mestre em Ciências da Saúde - UFT. Professor Assistente do Curso de Psicologia do Centro Universitário UnirG.

3Psicólogo. Mestre em Ciências da Saúde - UFT. Professor Assistente do Curso de Psicologia do Centro Universitário UnirG.

4Psicóloga. Especialista em Psicologia Clínica pelo Centro Universitário UnirG.

5Acadêmica do Curso de Psicologia do Centro Universitário UnirG, bolsista PIBIC Propesq 
a propensão à dependência da internet em universitários de Gurupi - TO. Metodologia: pesquisa quantitativa, na qual foram entrevistados 363 acadêmicos dos 15 cursos oferecidos em instituição de ensino superior empregando o instrumento Internet Addiction Test - IAT" (Teste de Dependência de Internet). Resultados: os sujeitos classificados como usuários normais ocuparam a porcentagem de $17 \%(n=61)$, enquanto os dependentes leves ocuparam 60\% ( $n=216)$, os dependentes moderados $22 \%(n=81)$ e os dependentes considerados como usuários graves representaram $1 \%$ da amostra $(n=5)$. Conclusão: Os resultados apresentaram a existência de uma dependência leve, seguido dos dependentes moderados.

Palavras chave: Usuários. Dependência. Tecnologia.

\section{PROPENSITY TO INTERNET DEPENDENCE: A STUDY WITH STUDENTS OF A UNIVERSITY CENTER}

\section{ABSTRACT}

Introduction: New technologies used to facilitate the way people communicate has been steadily increasing in the last decade. In line with this global amplitude growth, the excessive use of the phenomenon of the Internet to social networks has caused severe changes in society, as well as changes in the habits and behavior of individuals, which may characterize a dependency scenario. Objective: to investigate the propensity for Internet addiction among university students in Gurupi city. Methodology: quantitative research, in which 366 students from the 15 courses offered at a higher education institution were interviewed using the Internet Addiction Test (IAT). Results: Subjects classified as normal users occupied a percentage of $17 \%(n=61)$, while mild dependents occupied 
$60 \%(n=219)$, moderately dependent $22 \%(n=81)$, and dependents considered as serious users Represented $1 \%$ of the sample $(n=5)$. Conclusion: The results showed a mild dependence, followed by moderate dependents.

Keyords: Users. Dependency. Technology. 


\section{INTRODUÇÃO}

A internet é um grande conjunto de redes de comunicações acessada por bilhões de usuários no mundo inteiro, tendo dentre as suas vantagens possibilitar a comunicação e a obtenção de informações (OLIVEIRA \& PASQUALINI, 2014).

A tecnologia relacionada ao emprego de computadores e da internet, embora pareça recente, vem há mais de cinquenta anos sendo desenvolvida. Todavia, no intuito de agilizar a comunicação, este fenômeno emergiu e trouxe consigo tamanhas contribuições, como a rapidez e precisão nas informações acessadas e transmitidas. Os primeiros computadores surgiram por volta de 1945, sendo seu uso reservado para fins militares no auxílio nos cálculos científicos e, posteriormente, a partir da década de 60, seu uso tornou-se comum para uso civil. No entanto, conforme Levy (2005), naquela época não se previu que a disseminação do movimento de virtualização da informação e da comunicação iria atingir de modo tão significativo a vida social, bem como que os usuários adentrariam com facilidade no mundo cibernético.

Estudo realizado em 2012 assinalou que na época contava-se com aproximadamente mais de dois bilhões de usuários de internet, um número que está em constante crescimento, aproximadamente, meio milhão a cada dia. Os números de usuários de internet móvel quase dobraram do ano de 2011 para 2012, $99,8 \%$ dos assinantes dos planos de acesso à rede são referentes à internet móvel, representando 40 milhões de novos usuários, enquanto na telefonia fixa são apenas 16 milhões de pessoas (DAL CIN; MELO, 2013).

De acordo com King, Nardi e Cardoso (2014), o modo de vida das pessoas e a dinâmica social estão se modificando com o emprego de novas tecnologias digitais, bem como o acesso da população a internet, smartphones e redes sociais. Não se podem negar os benefícios como facilitar a comunicação, informação, retomar contato com pessoas distantes, agilizar o processo de trabalho entre outros, no entanto, o uso 
abusivo das tecnologias ganha novos adeptos diariamente de maneira silenciosa e preocupante, na qual indivíduos relegam suas relações pessoais, trabalho e estudos a um segundo plano para continuarem se mantendo "conectados".

Dentre as tecnologias de informação e telecomunicação que apresentam maior impacto social, destacam-se àquelas relacionadas à internet e à telefonia celular, as quais convergem ao se complementarem, gerando espaços de vida alternativos aos convencionais. Contudo, estudos recentes consideram que existem diferenças importantes no que se refere aos impactos sociais e psicológicos que a Internet e os celulares vêm causando (NICOLACI-DA-COSTA, 2004).

Atualmente, com a gama de informações providas pela facilidade e rapidez no acesso à internet, percebese constante transformação vida diária das pessoas. Tal fato pode ser evidenciado quando se faz referência aos benefícios e malefícios oriundos do fenômeno em questão, podendo afetar significativamente a rotina dos que utilizam demasiadamente esse recurso.

Em consonância ao exposto, dentre as finalidades destinadas às atividades realizadas na internet, temse destacado o crescimento acelerado da comunicação; da busca de informações e lazer; dos negócios financeiros e, somente depois, o uso destinado para fins educativos (DAL CIN, 2013).

Sobre a magnitude dos impactos revolucionários a dimensão social dos usuários da internet, é relevante apontar que ela está significativamente arraigada na sociedade contemporânea a ponto de ser concebida como algo necessário para o desempenho das mais variadas atividades, como econômicas, políticas, educacionais, entre outras (ABREU et al., 2008; YOUNG, 2009; DAL CIN, 2013).

Consequente ao crescimento acelerado e muitas vezes desproporcional do uso da internet, estudos sobre a dependência da mesma vem ganhando espaço nos bancos de dados científicos em todo mundo através de publicações sobre a sua utilização e finalidade. Ainda, o emprego crescente da internet tem tornado a linha divisória entre recreação e patologia cada vez mais tênue, ao ponto de constantemente impor um desafio ao profissional de 
saúde mental (CONTI et al., 2012).

Seja dentro das casas, do trabalho ou mesmo das universidades, homens e mulheres de todas as idades experimentam 0 fascínio da interatividade sem pudores e sem julgamentos externos proporcionados pela internet. A descoberta do novo, da sensação indescritível de liberdade, a negação do eu, a criação de uma identidade virtual, o anonimato e a facilidade de se relacionar estão entre os fatores que atraem milhões de usuários para os computadores (SA, 2012).

Em contrapartida, quando ocorrem prejuízos na vida social e no trabalho devido ao uso excessivo da internet, aumento do tempo conectado, bem como esforços empreendidos sem resultado para diminuir esse tempo, em que indivíduo poderá apresentar grande irritabilidade e/ou depressão, são alguns dos critérios de diagnóstico para dependência de internet (ABREU et al., 2013).

Nomofobia, este tem sido o termo utilizado para designar o desconforto ou angústia causados pelo medo de ficar incomunicável ou pela impossibilidade de comunicação por intermédio do telefone celular, computador ou internet (ficar off-line) (KING; NARDI; CARDOSO, 2014).

Ao abordar a temática dependência de internet, Abreu et al. (2008) afirmam que são variados os termos utilizados para definir o uso abusivo de computadores na literatura, como Internet Addiction, Pathological Internet Use, Internet Addiction Disorder, Compulsive Internet Use, Computer Mediated Communications Addicts, Computer Junkies e Internet Dependency. Essa pluralidade de definições se dá, fundamentalmente, em função das diferentes áreas de atuação dos profissionais que buscam compreendê-la. São opiniões que derivam de clínicos, pesquisadores, mídia, juristas, entre outros, nas quais são levados em consideração diferentes aspectos desses comportamentos na tentativa de contextualizá-los.

Conforme Young, Dong e Yin. (2011), os sintomas de dependência de internet mudam de acordo com 0 avanço e as transformações tecnológicas, e outro fator importante é o quanto essas tecnologias penetram a vida dos usuários que, às vezes, acabam por utilizar a internet de forma abusiva. Em outro estudo, Young 
(2012) assinala que ainda não há um padrão que defina as características dos dependentes, mas alguns tipos de comportamentos começam a ser comuns, como: o uso compulsivo da internet, uma preocupação constante em estar on-line, mentir ou esconder a extensão ou a natureza do seu comportamento on-line e uma incapacidade de controlar ou reduzir a quantidade de tempo que passa conectado.

Nesse sentido, uma gama de estudos apontam a necessidade de realização de novas pesquisas acerca da definição e caracterização do uso excessivo ou dependência de internet, a fim de auxiliar os profissionais da saúde mental a identificarem e buscarem recursos adequados para o quadro psíquico apresentado por muitos que buscam auxílio profissional. Portanto, a urgência da inclusão de critérios bem estabelecidos nos manuais diagnósticos e estatísticos de transtornos mentais (ABREU et al., 2008; YOUNG, 2012; CONTI et al., 2012).

Três abordagens conceituais marcam o início dos estudos visando desenvolver critérios diagnósticos para a dependência de internet.
Inicialmente, Griffiths (1999) descreveu tal dependência como um vício comportamental. Em um segundo momento, o modelo cognitivo comportamental assinalou o impacto dos pensamentos de um indivíduo em desenvolvimento de comportamentos desadaptativos, caracterizando a dependência de internet como generalizada quando ocorre o uso excessivo multidimensional dessa, e específica quando a dependência é especificamente em função da internet (DAVIS, 2001). Uma terceira abordagem considera que a dependência de internet seja classificada como um transtorno do controle dos impulsos (SHAPIRA et al., 2003).

Ademais, de acordo com pesquisas recentes (YOUNG, DONG, YING, 2011; YOUNG, 2012), deve ser considerado que os sintomas de dependência de internet mudam com o avanço das transformações tecnológicas, sendo aspecto relevante o quanto essas tecnologias penetram a vida dos usuários que, às vezes, acabam por utilizar a internet de forma abusiva.

Para Young (2012), mais importante do que o tempo que 0 
usuário passa conectado é o que a internet representa para sua vida e como lida com ela, sendo que para dependência é necessário haver prejuízo na vida social, laboral ou acadêmica do indivíduo afetado.

Em 2010, o Centro Internacional de Mídia e Agenda Pública efetuou pesquisa com mil universitários da Argentina, Chile, China, Eslováquia, Estados Unidos, Líbano, México, Reino Unido e Uganda, após responderem questionário on-line SurveyMonkey® esses foram convidados a executar a não utilizar por 24 horas quaisquer meios de tecnologias de informação e comunicação, como internet, TV, rádio, revistas ou jornais, celular, música, filmes, jogos etc. Transcorridas 24 horas os universitários deveriam escrever sobre a experiência. Como resultados, destacou-se as repetidas referências dos termos: vício, solitários, ansiosos, inquietos, chateados, loucos, vazio, pânico, estressados e desesperados (INTERNATIONAL CENTER FOR MEDIA AND THE PUBLIC AGENDA, 2010).

Dados como esses sugerem a proporção de espaço e importância que a internet vem ocupando na vida dos universitários, tanto para estudos, visto que atualmente é praticamente inconcebível não se pensar no uso da internet, quanto para demais atividades do dia a dia como relações sociais.

Entre os estudantes e, em especial os universitários, pesquisas de representatividade foram realizadas na Ásia pelo fato de ser um continente que possui países populosos aos quais os avanços tecnológicos emergem para o mundo e assinalam a elevada propensão apresentada por essa população à dependência da internet (YEN, YEN, KO, 2010). No entanto, por se tratar de uma temática recente, ainda é limitado o número de estudos sobre a mesma, o que reforça a necessidade de investigações sobre a prevalência da dependência de internet em universitários.

Desta forma, ao se deparar com esta realidade, o estudo em questão objetivou investigar a propensão à dependência da internet nos acadêmicos do Centro Universitário UnirG. Partiu-se do pressuposto que os acadêmicos diariamente destinam elevado número de horas dedicadas ao uso da internet, então estão propensos ao risco da dependência da mesma. 


\section{MATERIAL E MÉTODOS}

Este estudo compreende 0 desenvolvimento de uma pesquisa quantitativa descritiva. Para a sua realização, primeiramente foi necessário autorização juntamente com a Reitoria da IES e levantamento junto à Secretaria Geral Acadêmica da instituição, a fim de obter o quantitativo de alunos matriculados na IES. Constatou-se que no semestre de realização da pesquisa 0 centro universitário contava com 3953 (três mil novecentos e cinquenta e três) alunos distribuídos nos 15 cursos ofertados. Se tratando então de uma população finita, com o objetivo de maximizar a representatividade da amostra, a fórmula proposta por Barbetta foi utilizada para o cálculo amostral. Para tal cálculo, ficou estabelecida a frequência de dependência da internet de acordo com Oliveira e Pasqualini (2014), 45,5 (quarenta e cinco e meio), um nível de confiança de 95\% (expresso em números de desvio padrão) e um erro de até 5\%. Desta forma obteve-se uma amostra de 363 alunos, posteriormente estratificada por cursos, e submetida a uma seleção aleatória por sorteio.
A pesquisa foi aprovada pelo Comitê de Ética e Pesquisa com Seres Humanos, conforme a resolução 466/2012 do Conselho Nacional de Saúde, com número do Certificado de Apresentação para Apreciação Ética (CAAE) 53301215.1.0000.5518.

A coleta de dados ocorreu nas salas de aula da IES, durante uma pausa nas aulas combinadas previamente com os professores de cada curso, sendo apresentada a proposta da pesquisa e posteriormente convite para participação da mesma. Após 0 aceite da pesquisa 0 participante recebeu o TCLE e após a assinatura realizou a aplicação do instrumento Internet Addiction Test IAT elaborado por Kimberly Young e traduzido para o português por Conti e colaboradores (2012) , o qual pode ser visualizado no Quadro 1. O mesmo consiste em 20 itens de autopreenchimento com as respostas dadas em uma escala Likert de pontos, variando de 1 (raramente) a 5 (sempre), além do item 0 (não se aplica). Quanto maior sua pontuação, maior o grau de severidade da dependência. $O$ teste foi elaborado para avaliar quais áreas da 
vida de um indivíduo pode ser afetada por seu uso excessivo da internet. Seu estudo original elaborado por Widyanto e McMurran (2004) apresentou a análise fatorial, a consistência interna e as correlações das seis subescalas relacionando-as à idade e ao uso da internet. Esses incluíam: saliência (itens $10,12,13,15,19)$, uso excessivo (itens 1, 2, 14, 18, 20), abandono do trabalho (itens $6,8,9$ ), antecipação (itens 7,11 ), falta de controle (itens 5 , 16 e 17) e abandono da vida social (itens 3 e 4) (CONTI et al., 2012). Ressalta-se que das questões, a de número 7 (verificação do correio eletrônico), foi adaptada para verificação de e-mail e redes sociais, devido ao teste ter sido elaborado há alguns e ser considerado mais atual inserir tais meios. Além das 20 questões, também foi necessária uma breve identificação, compreendendo sexo, idade e curso de graduação cursado. Cabe ressaltar que ao final da aplicação foi disponibilizado o link (http://dependenciadeinternet.com.br/t este.php) para acesso ao questionário, bem como o crivo de correção, como forma de acesso individual aos resultados.

Quadro 1 - Questões do instrumento Internet Addiction Test

\footnotetext{
1. Está ligado à Internet mais tempo do que pretendia?

2. Com que frequência você abandona as tarefas domésticas para passar mais tempo na internet?

3. prefere a emoção da internet à intimidade com seu/sua parceiro(a)?

4 Com que frequência você cria relacionamentos com novo(a)s amigo(a)s da internet?

5. Com que frequência outras pessoas em sua vida se queixam sobre a quantidade de tempo que você passa na internet?

6. Com que frequência suas notas ou tarefas da escola pioram por causa da quantidade de tempo que você fica na internet?

7. Com que frequência você acessa seu e-mail antes de qualquer outra coisa que precise fazer?

8. Com que frequência piora o seu desempenho ou produtividade no trabalho por causa da internet?

9. Com que frequência você fica na defensiva ou guarda segredo quando alguém lhe pergunta o que você faz na internet?
} 
10. Com que frequência você bloqueia pensamentos perturbadores sobre sua vida pensando em se conectar para acalmar-se?

11. Com que frequência você se pega pensando em quando vai entrar na internet novamente?

12. Com que frequência você teme que a vida sem a internet seria chata, vazia e sem graça? 13. Com que frequência você explode, grita ou se irrita se alguém o(a) incomoda enquanto está na internet?

14. Com que frequência você dorme pouco por ficar conectado(a) até tarde da noite?

15. Com que frequência você se sente preocupado(a) com a internet quando está desconectado(a) imaginando que poderia estar conectado(a)?

16. Com que frequência você se pega dizendo "só mais alguns minutos" quando está conectado(a)?

17. Com que frequência você tenta diminuir o tempo que fica na internet e não consegue?

18. Com que frequência você tenta esconder a quantidade de tempo em que está na internet?

19. Com que frequência você opta por passar mais tempo na internet em vez de sair com outras pessoas?

20. Com que frequência você se sente deprimido(a), mal-humorado(a) ou nervoso(a) quando desconectado(a) e esse sentimento vai embora assim que volta a se conectar à internet?

Fonte: elaborado por Kimberly Young e traduzido por Conti et al. (2012)

Para análise dos dados foi utilizada a estatística descritiva simples, (frequência e porcentagem) através do software SPSS - Statistical Package for the Social Sciences versão 20.0 para Windows. Os dados foram organizados e analisados em forma de gráficos, tabelas e pela classificação de Young et al. (2011), sendo quantificados para obter a seguinte pontuação: no intervalo de 0-19 pontos foi classificado como um usuário normal, de 20-49 pontos foi classificado como dependente leve, de 50-79 pontos como dependência moderada e de 80-100 pontos como dependência grave de acordo com a classificação.

\section{RESULTADOS E DISCUSSÃO}


Participaram da pesquisa 363 acadêmicos dos 15 cursos da IES. Conforme explicado na metodologia, o número de participantes por curso deuse com base no número de acadêmicos matriculados nesses, por isso a variação entre as amostras, como pode ser visualizado na Tabela 1, com maiores porcentagens em Medicina e Direito.

Tabela 1 - Número de participantes por curso

\begin{tabular}{ll}
\hline Curso & Amostra \\
\hline Administração & 14 \\
Ciências Contábeis & 24 \\
Ciências da Computação & 4 \\
Comunicação Social & 2 \\
Direito Matutino & 43 \\
Direito Noturno & 48 \\
Educação Física & 15 \\
Enfermagem & 33 \\
Engenharia Civil Noturno & 21 \\
Engenharia Civil Matutino & 14 \\
Farmácia & 13 \\
Fisioterapia & 13 \\
Sistemas para Internet & 2 \\
Letras & 3 \\
Medicina & 65 \\
Odontologia & 24 \\
Pedagogia & 7 \\
Psicologia & 18 \\
\hline TOTAL & $\mathbf{3 6 3}$ \\
\hline
\end{tabular}

Da população total da amostra enquanto os dependentes leves (Figura 1), os sujeitos classificados ocuparam $60 \% \quad(n=216)$, os como usuários normais ocuparam a dependentes moderados $22 \%(n=81)$ porcentagem de $17 \% \quad(n=61), \quad$ e os dependentes considerados como 
usuários graves representaram $1 \%$ da amostra $(n=5)$.

Os resultados apresentaram a prevalência de dependência leve, seguido dos dependentes moderados. Ainda, em consonância com os resultados da pesquisa, há de se considerar que a facilidade da comunicação e da troca de informações pode afetar significativamente a vida dos que utilizam esse recurso.

Resultados semelhantes foram verificados por Dal Cin (2013) em pesquisa com profissionais da
Tecnologia da Informação, com níveis de dependência leve (40\%), moderada $(16 \%)$ e grave (7\%), ficando a maioria (51\%) classificados como usuário comum. Nesse estudo, alguns respondentes justificaram o uso da internet relacionado ao trabalho e necessidade de manter-se atualizados e a maioria, ao saber do resultado do teste IAT, ficou surpresa, seja pelo fato de serem dependentes, seja pelo fato de acharem que a classificação seria mais grave.

Figura 1 - Percentual de acordo com a classificação

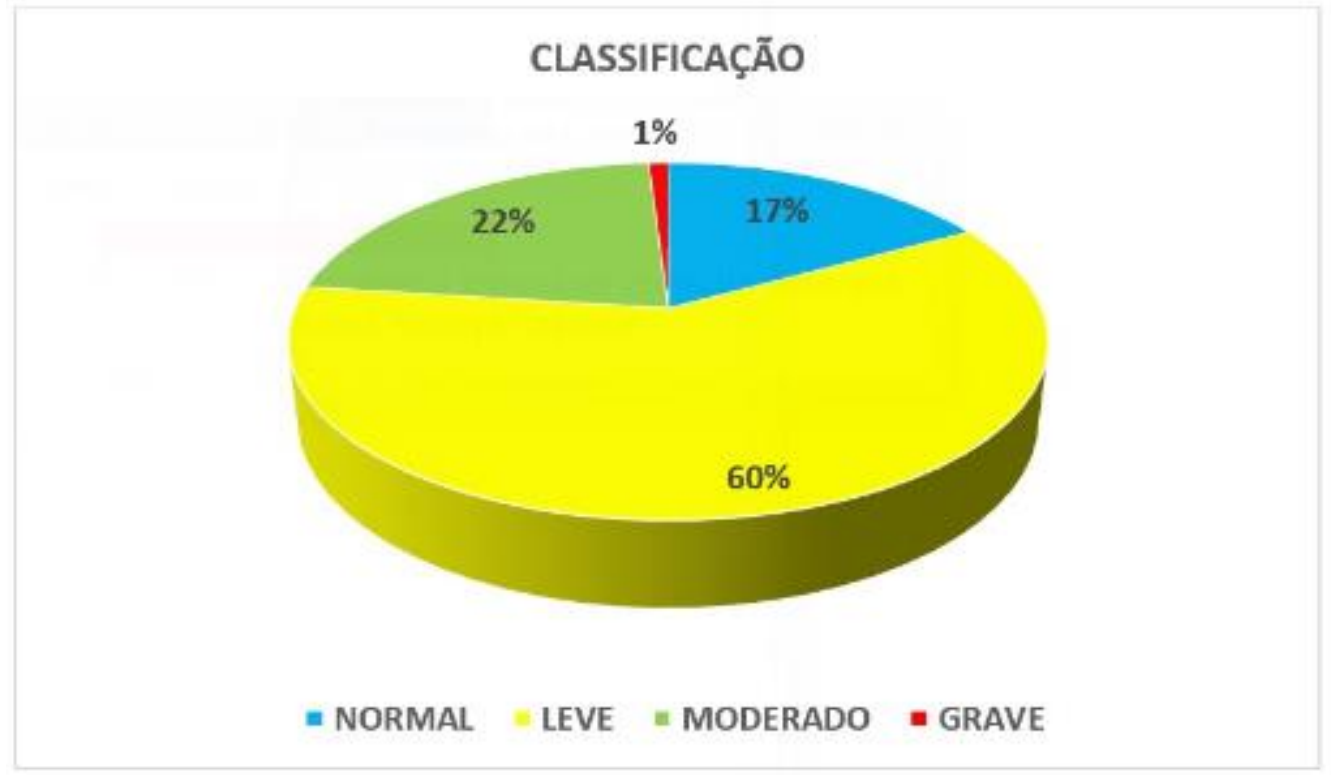

Corroborando, Oliveira e níveis leves e moderados de Pasqualini (2014) averiguaram em dependência da internet. Outro aspecto estudo realizado com 200 universitários importante assinalado por Dal Cin que $45,5 \%$ da amostra apresentou (2013) refere-se aos pesquisados 
omitirem informações importantes ao responder o questionário ou não perceber seu comportamento diante do mundo digital e talvez, pode-se até mesmo, incluir a percepção de terceiros, como as pessoas de convívio desse usuário.

De acordo com Young et al. (2011), a maioria dos usuários da internet possui algum poder de controle sobre o uso ou de alguma forma consegue reestabelecer tal domínio.

Em pesquisas recentes (YOUNG, DONG, YING 2011; YOUNG, 2012) é reforçado que os sintomas de dependência de internet mudam com o avanço e as transformações tecnológicas, sendo aspecto relevante o quanto essas tecnologias penetram a vida dos usuários que, às vezes, acabam por utilizar a internet de forma abusiva, aspecto ao qual deve-se atentar, bem como para a busca de medidas preventivas no intuito minimizar o referido fenômeno no meio acadêmico.

No que concerne à utilização das redes sociais, a incidência de dependência das mesmas para Pamoukaghlian (2011) não é tão alarmante como o vício em jogos na internet, contudo, o aumento no uso dessas redes vem trazendo problemas que interferem na vida dos que buscam empregá-la com o intuito de interação social. Ainda, esse novo e crescente interesse dos usuários da internet tem dificultado a elaboração de critérios diagnósticos consistentes para legitimar a dependência de internet como transtorno. Entretanto, estudos sugerem que a dependência em redes sociais pode ser um potencial problema de saúde mental para alguns usuários (KUSS; GRIFFITHS, 2011).

Ainda que mais da metade da amostra tenha sido classificada como leve, a influência da internet na sociedade como um todo, em especial entre adolescentes e jovens adultos já sugerem a necessidade de trabalhos voltados a prevenção de uma possível dependência. Neste sentido, acerca dos universitários, estes encontram-se entre a parcela da população que apresenta comportamentos que podem ser indicativos de dependência (ZBORALSKI, 2009; YEN, YEN, KO, 2010) 


\section{CONSIDERAÇÕES FINAIS}

O uso da internet a cada dia ganha mais espaço na vida das pessoas, seja por questões de trabalho, troca de informações ou estabelecimento de relações com velhos conhecidos distantes ou novos amigos. É inegável os benefícios proporcionados por essa tecnologia e praticamente impensável a vida sem a mesma, contudo, o seu uso indiscriminado pode trazer prejuízos à vida daqueles que não sabem administrar o tempo ou atividades realizadas on-line, sendo a população jovem e de universitários uma das mais afetadas.

Utilizando o IAT averiguou-se que, embora uma constante na vida dos universitários, por meio dos resultados os mesmos foram classificados como dependentes leves $(60 \%)$ e moderados (22\%), seguido dos normais (17\%) e apenas 5 participantes foram classificados como graves, representando $1 \%$ da amostra. Portanto, mesmo com as possibilidades do uso das tecnologias no cotidiano universitário, não foi constatada a dependência de internet.

Por fim, este estudo não implica um esgotamento de possibilidades acerca da dependência da internet em universitários, pois que esse é um campo atual e fértil para mais pesquisas, visto que as novas tecnologias tem sido cada vez mais frequentes no dia a dia das pessoas e os desdobramentos do seu uso vem chamando a atenção da sociedade.

\section{REFERÊNCIAS}

ABREU, C. N. et al. Dependência de Internet e de jogos eletrônicos: Uma revisão [Internet and videogame addiction: A review]. Revista Brasileira de Psiquiatria, v. 30, n. 2, p. 156-167, 2008. Disponível em: < http://www.scielo.br/pdf/rbp/v30n2/a14v30n2> Acesso em 08 set. 2016.

BRASIL. Conselho Nacional de Saúde. Resolução n. 466, de 12 de dezembro de 2012 que trata de pesquisas em seres humanos. Brasília: CNS; 2012. Disponível 
em: <http://conselho.saude.gov.br/resolucoes/2012/Reso466.pdf> Acesso em 06 Jun 2015.

CONTI, M. A. et al. Avaliação da equivalência semântica e consistência interna de uma versão em português do Internet Addiction Test (IAT). Rev Psiq Clín, v. 39, n. 3, p. 106-10, 2012. Dospinível em: < http://www.scielo.br/pdf/rpc/v39n3/a07v39n3> Acesso em 08 set. 2016.

DAL CIN, I. C. T. P. Dependência de Internet: Um Estudo com Profissionais e Estudantes da área de TI em Belo Horizonte. 2013. 170f. Dissertação (Mestrado em Administração). Faculdade Novos Horizontes, Belo Horizonte, 2001.

DAVIS, R. A. A cognitive behavioral modelo of pathological internet user. Computer in Human Bahavior, v. 17, n. 2, p. 187-195, 2001.

GRIFFITHS, M. Internet addiction: fact or fictions? Psychologist, v. 12, n. 5, p. 246250, 1999.

INTERNATIONAL CENTER FOR MEDIA AND THE PUBLIC AGENDA. The World Unplugged (Internet). College Park: University of Maryland, 2010. Disponível em: <http://theworldunplugged.wordpress.com>. Acesso em: 12 de dez. 2016.

KING, A. L. S.; NARDI, A. E.; CARDOSO, A. Nomofobia - Dependência do Computador e/ou Internet?. São Paulo: Atheneu, 2015. 352 p.

NICOLACI-DA-COSTA, A. M. Impactos psicológicos do uso de celulares: uma pesquisa exploratória com jovens brasileiros. Psic.: Teor. e Pesq., Brasília, v. 20, n. 2, p. 165-174, Aug. 2004 Available from <http://www.scielo.br/scielo.php?script=sci_arttext\&pid=S0102$37722004000200009 \& \operatorname{lng}=e n \& n r m=i s o>$. Acesso em 08 set. 2016. OLIVEIRA, F de; PASQUALINI, K. C. Os dependentes de internet no Brasil: realidade ou mito entre os universitários. Mimesis, Bauru, v. 35, n. 1, p. 95-140, 2014.

SA, G. M. À frente do computador: a Internet enquanto produtora de dependência e isolamento. Sociologia, Porto, v. 24, p. 133-147, dez. 2012. Disponível em: $<$ http://www.scielo.mec.pt/scielo.php?script=sci_arttext\&pid=S0872$4192012000200007 \&$ Ing=pt\&nrm=iso > Acessos em 08 set. 2016.

SHAPIRA, N. A. et al. Problematic internet use: proposed classification and diagnostic criteria. Depression and Anxiety, v. 17, n. 4, p. 2017-216, 2003. Disponível em: 
<http://onlinelibrary. wiley.com/doi/10.1002/da.10094/abstract;jsessionid=681C3EBB8 163EFC1FB6F86F7A3841E38.f03t03> Acesso em 10 dez. 2016.

YEN, C.; YEN, J.; KO, C. Internet addiction: ongoing research in Asia. World Psychiatry, v. 9, n. 2, p. 97, 2010.

YOUNG, K. S. Internet addiction: the emergence of a new clinical disorder. Cyberpsychol Behav, v.1, n. 3, p. 237- 44, 1998.

. Internet addiction: diagnosis and treatment considerations. J. Contemp. Psychother., v. 39, p. 241-246, 2009.

YOUNG, K.; DONG, Y.; YING, L. Estimativas de prevalência e modelos etiológicos da dependência de internet. In: YOUNG, K. S.; ABREU, C. N. Dependência de Internet: manual e guia de avaliação de tratamento. Porto Alegre: Artmed, 2011.

YOUNG, K. S. Compulsive Surfing. Netaddiction.com, 15 março 2012. Disponível em:http://www.netaddiction.com/index.php?option=com_content\&view=article\&id=62 \&It emid=85. Acessado em 25 Out 2016.

WIDYANTO, L.; MCMURRAN, M. Internet Addiction Test Research - Center for Internet Addiction. CyberPsychology \& Behavior. V. 7, n. 4, p. 443 - 450, 2004. Disponível em: <http://www.netaddiction.com/articles/InternetAddictionTestResearch.pdf> Acesso em 08 set. 2016..

Recebido em: 14/12/2016

Aprovado em: 01/06/2017 